Disclosure of Interests: Toshihisa Kojima Grant/research support from: Chugai Pharmaceutical (Investigator Initiated Study), Novartis, Nippon Kayaku, Eli Lilly, Eisai, Speakers bureau: Chugai Pharmaceutical, Takeda Pharmaceutical, Pfizer, Eli Lilly Japan, Bristol Myers Squibb, Ono Pharmaceutical, Daiichi Sankyo, Astelas, UCB, Janssen Pharmaceutical, Tanabe Mitsubishi, Masayo Kojima: None declared, Hajime Ishikawa: None declared, Sakae Tanaka Grant/research support from: KYOCERA Corporation and Asahi Kasei Corporation, Consultant for: Amgen Astellas BioPharma K.K., KYOCERA Corporation, Pfizer and Daiichi Sankyo Co., Ltd., Speakers bureau: Asahi Kasei Corporation, Astellas Pharma Inc, Ayumi Pharmaceutical Corporation, Eisai Co., Ltd., Ono Pharmaceutical Co., Ltd., Daiichi Sankyo Co., Ltd, Taisho Toyama Pharmaceutical Co. Ltd., Mitsubishi Tanabe pharma Corporation, Chugai Pharmaceutical Co. Ltd., Teijin Pharma Ltd., Eli Lilly, Hisamitsu Pharmaceutical Co, Inc. Pfizer, Bristol-Myers., Keiichiro Nishida: None declared, Nobuhiko Haga: None declared, Yukio Masaoka: None declared, Jun Hashimoto: None declared, Hisaaki Miyahara: None declared, Yasuo Niki: None declared, Tomoatsu Kimura: None declared, Hiromi Oda: None declared, Shuji Asa Speakers bureau: AbbVie, Astellas, Bristol-Myers Squibb, Chugai, DaiichiSankyo, Eisai, Janssen, Takeda, and UCB Japan, Koji Funahashi: None declared, Naoki Ishiguro Grant/research support from: AbbVie, Asahi Kasei, Astellas, Chugai, Daiichi-Sankyo, Eisai, Kaken, Mitsubishi Tanabe, Otsuka, Pfizer, Takeda, and Zimmer Biomet, Consultant for: Ono, Speakers bureau: Astellas, Bristol-Myers Squibb, Daiichi-Sankyo, Eli Lilly, Pfizer, and Taisho Toyama

DOI: 10.1136/annrheumdis-2019-eular.1689

\section{AB1323 OSTEOPHYTOSIS PROCESS SUGGEST INVOLVEMENT OF MAST CELLS, REVEALED BY WHOLE TRANSCRIPTOME ANALYSIS OF OSTEOPHYTES FROM KNEE OSTEOARTHRITIS PATIENTS}

Priya Kulkarni ${ }^{1}$, Sulev Koks ${ }^{1}$, Abhay Harsulkar ${ }^{2}$, Aare Martson ${ }^{3} .{ }^{1}$ University of Tartu, Department of Pathophysiology, Tartu, Estonia; ${ }^{2}$ Bharati Vidyapeeth University, Department of Pharmaceutical Biotechnology, Pune, India; ${ }^{3}$ University of Tartu, Department of Traumatology and Orthopedics, Tartu, Estonia

Background: Cartilage-loss is a central pathology of osteoarthritis while synovitis, sub-chondral bone sclerosis and osteophytes (OFs) formation are counted as integral phenomenon. Osteophytosis is a process of OFs formation, in which mesenchymal stem cells from periosteum and synovium, along with a number of cytokines, growth factors and biochemical factors gives out bony out-growth at margin of the affected joint. Although frequently associated, OFs are one of the less focused areas in OA research, particularly from the molecular mechanism point of view, involved in their formation. Also, it is a good ambiguity if OFs is a pathological condition linked with altered biochemical milieu or a functional adaptation for the joint instability.

Objectives: Accounting its importance in clinical $O A$ and the obscure biochemical background, this study strives for a deeper comprehension of molecular mechanism and active pathways, involved in OF formation Methods: We performed whole transcriptome analysis of OFs, collected from six knee OA patients during knee replacement surgery. Non-OF tissue from tibial plateau of the same patient was used as a control. After RNA isolation, RNA-seq was performed using SOLiD 5500W platform and fragment sequencing chemistry. For differential gene expression analysis, non- normalized raw counts were used for the EdgeR package. To find differential transcriptome between OF and control, we used group-wise comparisons where negative binomial fitting was followed by exact test. Finally, RT-PCR of significantly expressed genes was run for trascriptome validation.

Results: After cluster analysis, total 597 genes were significantly expressed between OFs and controls out of which, 323 genes were seen up-regulated ( $\operatorname{LogFC} \geq 2)$ while 274 were down-regulated (LogFC $\leq$ 2). Further, 88 genes showed marked increase $(P<0.01)$; here CPA3, Selectin E, MS4A2, PLA2G2A and CSN1S1 were prominent. Similarly, 23 genes were with a noteworthy down-regulation $(P<0.01)$ and $A P O B$, CADM2, TNEFF2, GNAZ and GABRA2 remained as first five. GO analysis of the best extracted genes indicated significant involvement of ECM proteoglycan, GABA A receptor pathway (Reactome 2016 - homo sapiens_R-HSA-3000178 and R-HSA-977441, respectively), osteoblast and TNF- $\alpha$ signalling pathways (WikiPathways 2016 -homo sapiens WP322 and homo sapiens WP231 respectively). Finally, tracsriptome results were validated using RT-PCR of significantly expressed genes (CPA3, MMP-1, 3 and 13).

Conclusion: Significant expression of CXCL9, 10, 11 and PGR4 indicate chemotaxis and immune cells deployment; outstanding CPA3 and MS4A2 indicate active involvement of mast cells. Accounting a previous knowledge that 'mast cell products' are linked with increased osteoblastic and osteoclastic activity and impose pathological changes in articular and periarticular tissue, marked expressions of MC products or mediators here, enable us to propose an association between MCs and OF. Presence of E-selectin on endothelial cell surface is known to promote adhesion of neutrophils and further release of TNF $\alpha$ and IL1. Expression of PLA2G2A suggests increased prostaglandin synthesis and indicates activation of inflammatory cascade. In conclusion, this transcriptome analysis suggests a significant association of mast cells with osteophytosis. The genes involving chemotaxis, degranulation, cytokinin synthesis mark the initiation of OF. On the other hand, proteases metrixremodelling enzymes may indicate their growth. A host of different signalling molecules denotes involvement of canonical and non-cannonical pathways that warrants further investigations.

Disclosure of Interests: None declared

DOI: 10.1136/annrheumdis-2019-eular.7318

\section{AB1324 MEASUREMENT PROPERTIES OF THE 10-ITEM CONNOR-DAVIDSON RESILIENCE SCALE AMONG PATIENTS WITH TOTAL KNEE REPLACEMENT BASED ON ITEM RESPONSE THEORY}

Ka Keat Lim', Yu Heng Kwan ${ }^{1}$, Seng Jin Yeo², William Yeo ${ }^{3}$, Julian Thumboo ${ }^{4,5}$, David Matchar ${ }^{1,6}$, Ying Ying Leung ${ }^{4,5}$. 'Duke-NUS Medical School, Programme in Health Services and Systems Research, Singapore, Singapore; ${ }^{2}$ Singapore General Hospital, Department of Orthopaedic Surgery, Singapore, Singapore; ${ }^{3}$ Singapore General Hospital, Orthopaedic Diagnostic Centre, Department of Orthopaedic Surgery, Singapore, Singapore; ${ }^{4}$ Singapore General Hospital, Department of Rheumatology and Immunology, Singapore, Singapore; ${ }^{5}$ Duke-NUS Medical School, Singapore, Singapore; ${ }^{6}$ Duke University Medical Center, Department of Medicine (General Internal Medicine), Durham, United States of America

Background: The number of older adults undergoing total knee arthroplasty (TKR) for knee osteoarthritis (OA) has been increasing. While psychological resilience may have a role in outcomes after TKR[1], no psychological resilience scale has been validated among patients with knee OA.

Objectives: To examine the measurement properties of the 10-item Connor-Davidson Resilience Scale (CD-RISC10) among older adults with knee $\mathrm{OA}$

Methods: Using data from 700 consecutive consented patients with knee OA enlisted for TKR, we examined four measurement properties of CDRISC10. For structural validity, we fitted graded response model (grm) and examined standardized root-mean square residual (SRMR) for unidimensionality, Chi-square p-value for item fit, residual correlation for local independence and item-characteristic curves for monotonicity. For internal consistency, we examined Cronbach's alpha. For construct validity, we hypothesized that CD-RISC10 as a measure of psychological resilience would be moderately correlated (Pearson's R $0.30-0.50$ ) with self-efficacy and mental well-being but weakly correlated (Pearson's $R<0.30$ ) with physical and social well-being (Table 1). For measurement invariance, we examined uniform and non-uniform differential item functioning (DIF) between gender, age group ( $\leq$ median 66 vs $>66$ years), and language (English vs Chinese) and reported the McFadden's $R^{2}$. We used mirt and lordif packages on Rx64 v3.5.1 to examine grm and DIF respectively. We reported the findings based on COnsensus based Standards for the selection of health Measurement Instruments (COSMIN)[2].

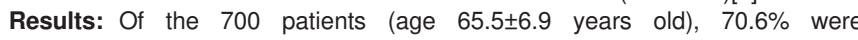
females, $83.9 \%$ Chinese, $55 \%$ responded questionnaire in Chinese. Mean CD-RISC10 score was $27.5 \pm 5.2$, with minimal floor $(0.1 \%)$ and ceiling effects (3.9\%). CD-RISC10 demonstrated unidimensionality (SRMR=0.053) local independence (residual correlation=-0.025), monotonicity (adequate looking item characteristic curves) and internal consistency (Cronbach's alpha $=0.91$ ). Items 1 and 4 had potential misfit (Chi-square $p>0.01$ ). All a-priori hypotheses were met (Table 1). No item had significant DIF with age, gender and language, except item 4 had borderline non-uniform DIF across language (McFadden's $R^{2}=0.03$ ).

Conclusion: We demonstrated structure validity, internal consistency, construct validity and measurement invariance of CD-RISC10 in the measurement of psychological resilience among older adults with knee OA.

\section{REFERENCES}

[1] Rebagliati, et al. Funct Neurol, 2016. 31(3): 171-7.

[2] Mokkink, et al. COSMIN User Manual. 2018 
Table 1. Hypothesized and actual correlations for convergent validity

\begin{tabular}{|c|c|c|c|c|c|}
\hline & \multicolumn{2}{|c|}{ Hypothesized } & \multicolumn{2}{|c|}{ Actual } & \multirow{2}{*}{$\begin{array}{c}\text { Hypotheses } \\
\text { Met }\end{array}$} \\
\hline & Direction & $\mathbf{R}$ & $\mathbf{R}$ & $\begin{array}{c}\mathrm{p}- \\
\text { value }\end{array}$ & \\
\hline \multicolumn{6}{|l|}{ Internal resource } \\
\hline Pain self-efficacy & + & $0.30-0.50$ & 0.48 & 0.000 & Yes \\
\hline \multicolumn{6}{|l|}{ Mental well-being } \\
\hline HADS anxiety & - & $\begin{array}{c}-0.30-- \\
0.50\end{array}$ & -0.37 & 0.000 & Yes \\
\hline HADS depression & - & $\begin{array}{c}-0.30-- \\
0.50\end{array}$ & -0.31 & 0.000 & Yes \\
\hline SF-36 mental health & + & $0.30-0.50$ & 0.30 & 0.000 & Yes \\
\hline \multicolumn{6}{|l|}{ Physical well-being } \\
\hline WOMAC physical function & - & $>-0.30$ & -0.17 & 0.000 & Yes \\
\hline WOMAC pain & - & $>-0.30$ & -0.16 & 0.000 & Yes \\
\hline WOMAC stiffness & - & $>-0.30$ & -0.11 & 0.005 & Yes \\
\hline SF-36 physical functioning & + & $<0.30$ & 0.15 & 0.000 & Yes \\
\hline \multicolumn{6}{|l|}{ Social well-being } \\
\hline $\begin{array}{l}\text { Lubben's social network } \\
\text { score }\end{array}$ & + & $<0.30$ & 0.22 & 0.000 & Yes \\
\hline
\end{tabular}

HADS:Hospital Anxiety \& Depression Scale; SF-36:Short-Form 36; WOMAC:Western Ontario \& McMaster Universities Osteoarthritis Index

Disclosure of Interests: Ka Keat Lim: None declared, Yu Heng Kwan: None declared, Seng Jin Yeo: None declared, William Yeo: None declared, Julian Thumboo: None declared, David Matchar: None declared, Ying Ying Leung Grant/research support from: Abbvie, Novartis, Speakers bureau: Abbvie and Novartis, Speakers bureau: Novartis DOI: 10.1136/annrheumdis-2019-eular.1014

\section{AB1325 HAND FUNCTION IS IMPAIRED IN PATIENTS WITH RHEUMATOID ARTHRITIS, PSORIATIC ARTHRITIS, AND PSORIASIS COMPARED TO HEALTHY CONTROLS}

Anna-Maria Liphardt ${ }^{1}, 2$, Sonja Liehr ${ }^{1}$, Eva Manger ${ }^{1}$, Lisa Bieniek ${ }^{1}$, Arnd Kleyer ${ }^{1}$, David Simon ${ }^{1}$, Koray Tascilar ${ }^{1}$, Michael Sticherling ${ }^{3}$, Jürgen Rech ${ }^{1}$, Georg Schett ${ }^{1}$, Axel Hueber ${ }^{1,4}$. ${ }^{1}$ Friedrich-Alexander-University Erlangen-Nuremberg, University Hospital Erlangen, Internal Medicine 3 - Rheumatology and Immunology, Erlangen, Germany; ${ }^{2}$ German Sport University Cologne (DSHS Köln), Biomechanics and Orthopaedics, Köln, Germany; ${ }^{3}$ Friedrich-Alexander-University Erlangen-Nuremberg, Dermatology, Erlangen, Germany; ${ }^{4}$ Sozialstiftung Bamberg, Rheumatology, Bamberg, Germany

Background: Inflammatory-rheumatic diseases affect hand joints with swelling and pain, leading to joint destruction. Even patients with psoriasis only may suffer subclinical inflammation in the hand joints, leading to changes of bone [1]. Finger joint involvement is different for rheumatoid arthritis (RA), psoriatic arthritis (PsA) and psoriasis (Pso) but hand function in these patients is not well characterized.

Objectives: To quantify and compare grip strength and hand function in patients with RA, PsA and Pso and to relate these outcomes to disease activity.

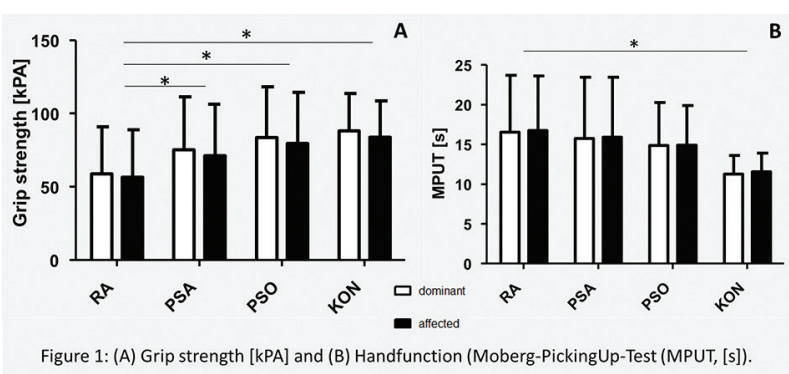

Figure 1

Methods: Patient diagnosed with RA (ACR/EULAR 2010), PsA (Caspar) and Pso and 54 healthy individuals were included in the study after written informed consent. Maximal isometric grip strength (kPA) was measured with a hand dynamometer (Lafayette Instrument, Lafayette, IN, USA) as the highest value out of three measurements. Hand function was determined by way of the Moberg Picking-Up Test (MPUT) and the fastest (s) of two measurements was used. Disease activity was calculated as DAS28_ESR and TJC/SJC 78/76 was recorded. One-way Analysis of Variance (ANOVA), factorial ANOVA and Games-Howell post-hoc testing was performed $(p<0.05)$

Results: 101 RA (63 f; $38 \mathrm{~m}$; age: $59.1 \pm 13.2 \mathrm{yrs}$ ), 92 PsA (48 f, $44 \mathrm{~m}$; age $54.8 \pm 11.6 \mathrm{yrs})$ and 51 Pso patients (19 f, $32 \mathrm{~m}$; age 47.3 \pm 14.1 yrs), as well as 54 healthy control subjects ( $30 \mathrm{f}, 24 \mathrm{~m}$; age $54.6 \pm 16.5$ yrs) participated in the study. Disease duration (yrs) was not different between groups (RA: $11.03 \pm 10.1$, PsA: $9.1 \pm 9.75$, Pso: $12.5 \pm 11.7$; $\mathrm{p}=0.156$ ) but Pso patients in our cohort were younger compared to RA and PsA patients. Mean DAS28_ESR was < 3.2 for all patient groups with control subjects (DAS28 $1.6 \pm 0.7$ ) having lower scores compared to all patient groups and Pso patients presenting with lower DAS28 (2.3 $\pm 1.3)$ compared to RA $(3.0 \pm 1.3)$ and PsA (2.9 \pm 1.3$)$. While TJC was higher for all patients compared to controls (RA: $5.2 \pm 6.8$, PsA: $5.9 \pm 8.1$ Pso: $3.2 \pm 6.4$; CON: $0.5 \pm 1.4 ; p=0.00$ ), SJC was higher for RA and PsA compared to Pso and CON (RA: $0.7 \pm 1.0$, PsA: $0.9 \pm 2.2$ Pso: $0.1 \pm 0.3$; CON: $0.5 \pm 1.4 ; p=0.000)$. Grip strength of the dominant and/or affected hand of RA patients was lower compared to PsA, Pso and CON (Figure 1A). In contrast to this, hand function of the same hand in all tested patient groups was reduced compared to CON (Figure 1B). The results for grip strength and hand function were not different for the dominant and the affected hand and are independent of age differences between groups.

Conclusion: Overall, RA patients showed significantly lower grip strength compared to PsA and Pso patients as well as to the control group, whereas all three patient groups performed worse in the MPUT compared to the control cohort. This suggests that grip strength may be preserved longer than hand function in patients with inflammatory rheumatic disease. Further, it was surprizing that hand function was reduced in Pso patients without arthritic correlate compared to healthy subjects. This suggests that hand function may provide a potential parameter for discriminating Pso patients at risk for subclinical joint involvement.

This study was funded by research support from Novartis Pharma, $\mathrm{GmbH}$.

\section{REFERENCES}

[1] Faustini F, Simon D, et al. 2016, Ann Rheum Dis 75: 2068-2074.

Disclosure of Interests: Anna-Maria Liphardt Grant/research support from Novartis Pharma GmbH, Sonja Liehr: None declared, Eva Manger: None declared, Lisa Bieniek: None declared, Arnd Kleyer Grant/research support from: Lilly, Consultant for: Lilly, Speakers bureau: Abbvie, David Simon Grant/research support from: Novartis, Consultant for: Lilly, Speakers bureau: Janssen, Koray Tascilar: None declared, Michael Sticherling: None declared, Jürgen Rech Grant/research support from: Bristol-Myers Squibb and Celgene (greater than $\$ 10,000$ ), Consultant for: Bristol-Myers Squibb, Celgene, Chugai, GlaxoSmithKline, Janssen, Eli Lilly, Novartis, Roche, Sanofi Aventis, and UCB (in total more than $\$ 10,000$ ), Speakers bureau: Bristol-Myers Squibb, Celgene, Chugai, GlaxoSmithKline, Janssen, Eli Lilly, Novartis, Roche, Sanofi Aventis, and UCB (in total more than $\$ 10,000$ ), Georg Schett: None declared, Axel Hueber Grant/research support from: Novartis, Pfizer, Consultant for: Lilly, Speakers bureau: Lilly, Novartis, Janssen, Abbvie

DOI: 10.1136/annrheumdis-2019-eular.4733

\section{AB1326 INCREASED FIBRINOGEN TO ALBUMIN RATIO IN ANKYLOSING SPONDYLITIS: CORRELATION WITH DISEASE ACTIVITY}

Meng Liu, Yukai Huang, Zhengping Huang, Qidang Huang, Xin Guo, Yunqing Wang, Wengming Deng, Zhi-Xiang Huang, LI Tian-Wang. Guangdong Second Provincial General Hospital, Department of Rheumatology and Immunology, Guangzhou, China

Background: Fibrinogen to albumin ratio (FAR) has emerged as a new effective biomarker which can reflect the severity of chronic inflammation. However, none of study has focused on the role of FAR in ankylosing spondylitis (AS).

Objectives: The study aimed to investigate the association between FAR and the disease activity of AS.

Methods: The retrospective study included 117 AS patients and 165 ageand gender-matched healthy controls. AS patients were divided into remission group (Bath Ankylosing Spondylitis Disease Activity Index 